\title{
Développement d'interfaces entre les services de santé publique et les services cliniques de première ligne à Québec : une étude de cas
}

\section{Developing Public Health - Primary Care Interfaces in Quebec: A Case Study}

\author{
买 \\ by JALILA JBILOU, MD, MSC \\ Département de médecine sociale et préventive \\ Université Laval, Laval, Québec. \\ DANIEL REINHARZ, MD, PHD \\ Associate Professor \\ Département de médecine sociale et préventive \\ Université Laval, Laval, Québec
}

\section{Résumé}

Les services de santé publique ont, entre autres, pour mission de développer des actions susceptibles de diminuer les inégalités en santé des différents groupes de la population. Pour pouvoir rejoindre ces groupes, ils ont à mettre en place des interfaces avec divers acteurs, dont ceux de la première ligne. La présente étude de cas vise 
l'identification, au moyen d'une analyse qualitative, des facteurs organisationnels facilitants et des barrières à l'établissement d'une interface entre les services de santé publique et ceux de première ligne dans un territoire de la région de Québec. Les résultats révèlent que les agences de santé publique doivent entreprendre des stratégies ciblées visant à favoriser l'appropriation de nouveaux concepts par les cliniciens tels que la perspective populationnelle, avant de songer aux structures les plus à mêmes de développer et formaliser cette interface.

\begin{abstract}
Public health agencies $(\mathrm{PH})$ have various functions. One of them is to develop initiatives that will help decrease health inequalities among different groups in the population. To reach vulnerable groups, $\mathrm{PHs}$ must establish interfaces with various players, including primary care clinicians (PCC). Through a qualitative analysis, this case study identifies the organizational factors that either help or hinder the establishment of a functional interface in an area of the Quebec City region. Findings indicate that PHs must first undertake specific strategies to implement a new paradigm (such as the population perspective) among clinicians before introducing structural changes for developing and formalizing PH-PCC interfaces.
\end{abstract}

\title{
Introduction
}

L'historique de la santé publique (SP) et celui de l'organisation des services cliniques de première ligne (SCPL) au Québec sont riches en réformes, législations et politiques publiques. Néanmoins, les structures implantées ont peu formalisé les conditions favorables au développement d'interfaces SP-SCPL. Ces deux secteurs ont, dans une large mesure, évolué parallèlement et séparément sans vraiment saisir les occasions d'influence mutuelle qui soffraient dans cet environnement de changement organisationnel.

Néanmoins, le rapprochement entre ces deux secteurs a été une préoccupation constante des gestionnaires et il repose sur deux justifications.

Premièrement, un intérêt mutuel d'aller rechercher chez l'autre, ce qui est nécessaire pour mieux répondre à sa mission. C'est ainsi qu'on demande dorénavant aux cliniciens détendre leur pratique à des activités qui englobent une expertise détenue par la SP : la prévention et la promotion de la santé (Commission sur l'exercice de la médecine des années 2000 1998), interventions $<<$ extra-curatives $>>$ pouvant avoir une influence sur la santé et le bien-être des patients atteints de pathologies complexes comme le diabète (Hawk et Lippman 2000; Visscher et Seidell 2001), les maladies cardiovasculaires (Eastcott 1982; Cameron et al. 2000; Hawk et Lippman 2000; 
Glick 2002) ou le cancer (Hawk et Lippman 2000; Adami et al. 2001; Visscher et Seidell 2001; Storm et Olsen 2002). Quant à la SP, elle a besoin de terrain pour pouvoir exercer certaines de ses fonctions essentielles (Lévesque et Bergeron 2003). Donc, une collaboration entre ces deux secteurs est souhaitable autant d'un point de vue clinique et économique, que paradigmatique, surtout dans des systèmes de santé qui privilègient l'intégration comme option pour mieux répondre aux divers besoins en santé de la population (Wright 1995; Grunfeld, Gray et al. 1999; Frumkin 2000; Elster et Callan 2002; Shi et al. 2002).

Deuxièmement, le rapprochement entre SCPL et SP découle de la responsabilité que se reconnaît l'État par rapport à l'amélioration de la santé de sa population. En accord avec les connaissances actuelles, la santé est vue comme résultante de nombreux déterminants, les services cliniques étant un parmi d'autres. Dans cette perspective, la SP, de par sa responsabilité populationnelle, est devenue un secteur légitimé pour mobiliser l'ensemble des ressources susceptibles d'améliorer la santé, tant intraqu'extra-sectorielles. Le maillage répond donc à un objectif fondamental de l'État.

Or, force est de constater que malgré les intérêts reconnus et les efforts déployés pour les rapprocher, ces deux secteurs continuent à fonctionner largement en silos (Lasker 1997; Brandt et Gardner 2000; Lasker et al. 2001). Le développement d'une interface SP-SCPL reste complexe. De plus, peu dans ce que rapporte la littérature aborde spécifiquement la question de la dynamique des acteurs et des facteurs tant facilitant quentravant la mise en place d'un arrimage fonctionnel entre SP et SCPL. Dans ce cadre, l'objectif de ce travail est d'identifier les facteurs organisationnels qui facilitent ou entravent la rédaction d'une proposition d'un plan d'organisation des services de santé en première ligne qui fait explicitement la promotion du développement d'interfaces SP-SCPL dans un territoire de la région de Québec.

\section{Méthodologie}

\section{Approche conceptuelle}

Deux cadres théoriques, testés par d'autres auteurs en analyse organisationnelle (Reinharz 1999; Reinharz et al. 2000; Jbilou 2004), sont repris dans ce travail :

1. Les archétypes (Hinings et Greenwood 1988) pour dresser un portrait organisationnel de l'interface SP-SCPL à des moments-clés de l'histoire récente du site étudié. Ce cadre propose deux composantes de l'organisation : la structure organisationnelle $(\mathrm{SO})$ et les schèmes interprétatifs $(\mathrm{SI})$. La SO sétudie à travers : les rôles et les responsabilités, les mécanismes de décision et les mécanismes de gestion des ressources humaines. Les SI se représentent par : le domaine d'activité 
occupé par l'organisation, les principes d'organisation (valeurs et idées qui soustendent la perception de comment l'organisation devrait être structurée) et les critères d'évaluation (mécanismes par lesquels une évaluation des activités du groupe est réalisée).

Ce cadre permet de décrire la stabilité des organisations à des moments-clés de leur évolution en positionnant les acteurs ou groupes d'acteurs les uns par rapport aux autres. Cette stabilité est étudiée à travers deux notions : la convergence et la cohérence. La convergence s'interprète au moyen de l'identification des similitudes et/ou différences qui existent entre les divers groupes d'acteurs. Elle sáanalyse autant sur le plan de la SO (ćest-à-dire une répartition claire et acceptée des rôles et des ressources entre les différents acteurs face à un objet) que des SI (c'est-à-dire une vision partagée sur les responsabilités de chacun face à un objet). La cohérence s'interprète au moyen de l'identification des similitudes et/ou différences existantes entre SO et SI au sein de chacun des groupes d'acteurs (ćest-à-dire la concordance entre ce que la structure prescrit en ce qui concerne les responsabilités et ce que les croyances et valeurs des acteurs supposent par rapport à un objet). En effet, l'organisation est considérée stable si, pour chacun des groupes d'acteurs, la SO est cohérente avec les SI dominants. Si les schèmes sont amenés à changer, suite à l'acquisition de nouvelle nouvelles données probantes scientifique, par exemple, la cohérence sestompe et l'organisation devient instable. Face à cette instabilité, les acteurs développent des stratégies pour amener l'organisation à évoluer vers une nouvelle configuration organisationnelle plus cohérente avec leurs nouveaux SI; ćest à ce moment quémerge une dynamique entre acteurs que nous analyserons au moyen de la théorie des coalitions.

2. La théorie des coalitions (Gamson 1961) pour décrire la dynamique qui sous-tend le développement des stratégies collectives des acteurs et pour identifier les leaders. Cette théorie cadre létude de la dynamique des acteurs sur trois dimensions : les transactions, les liens et les contrôles. Les transactions regroupent les bénéfices recherchés par les membres de la coalition et les ressources qu'ils y concèdent pour réaliser leurs objectifs. Les transactions sopèrent selon un principe d'une recherche d'efficience ou d'un gain supérieur à l'investissement consenti. La coalition permet ainsi l'obtention de bénéfices que l'individu seul ne peut avoir. Les liens sont fondés sur des préférences non utilitaires qui favorisent l'émergence de la coalition. Les liens se constituent autour de la notion d'appartenance qui est perçue par les acteurs et qui peut être idéologique (positionnement politique), corporative (corps professionnel ou syndical) ou même affective (lien d'amitié). Le contrôle est la capacité qu'a un acteur, individuel ou collectif, de conformer une décision de groupe à son propre choix. C'est cette dernière qui permet d'identifier le ou les leaders de la coalition. 
Finalement, et afin d'offrir une présentation structurée des conséquences de cette dynamique, nous avons utilisé une grille d'analyse basée sur les trois principales conséquences d'un changement organisationnel : les conséquences symboliques qui sont relatives à l'image véhiculée par la SO proposée, les conséquences substantives qui représentent les propositions concrètes mises de l'avant dans le rapport soumis et les conséquences politiques basées sur la redéfinition des rapports entre les acteurs concernés et leur évolution future (Greenwood et Hinings 1988; Friedberg 1993; Denis et al. 1996).

\section{Devis et collecte d'information}

Une étude de cas a été réalisée au moyen d'une approche qualitative. Elle a porté sur un territoire administratif de la région de Québec.

L'unité d'étude a été une table territoriale de concertation sur l'organisation des services (TTO) dans ce territoire. Les TTO sont issues d'une initiative régionale préconisée par le plan régional de transformation des services de première ligne. D'un point de vue chronologique, cette initiative précède légèrement la réforme que connaît présentement le système de santé québécois. Cependant, elle en partage les assises paradigmatiques, qui sont d'assurer, grâce à une mobilisation de l'ensemble des acteurs de 1ère ligne, une meilleure réponse globale aux besoins de la population. Cette initiative visait à organiser, dans chacun des territoires de la région, une meilleure interface entre les services offerts dans les cliniques privées et ceux offerts par le réseau institutionnel, afin de mieux répondre aux besoins non seulement des consommateurs de services, mais également des populations du territoire. Les TTO ont pour mandat de déposer, après un an de travaux, un rapport proposant un modèle d'organisation des services cliniques de première ligne. Au début, seuls les cliniciens participaient aux TTO. Cependant, certaines ont accepté la participation d'autres acteurs tels que la SP, les infirmières et des gestionnaires. La TTO étudiée fait partie de celles dites «mixtes».

Les personnes ressources ciblées ont été l'ensemble des participants à la TTO du territoire étudié, soit 15 individus. La provenance des répondants est représentative des différents groupes d'acteurs visés par le présent travail. De plus, ces répondants parlent au nom du groupe qu'ils représentent autour de la TTO. Ils ont été contactés par téléphone dans un premier temps. Suite à leur consentement de participer à l'étude, soit un taux de réponse de $100 \%$, un résumé du projet et les principaux items du questionnaire leur ont été envoyés. Toutefois, la saturation de l'information ayant été obtenue après 12 entrevues, il n'a pas été nécessaire de rencontrer les quatre autres membres. Ainsi, les répondants ${ }^{1}$ interviewés sont :

1 Le masculin a été adopté dans la présentation des répondants pour préserver leur anonymat. 
- un clinicien représentant l'Unité de médecine familiale (UMF);

- un médecin-conseil régional représentant la Régie régionale (RRSSS-03);

- un clinicien exerçant en clinique privée et représentant la Direction régionale de médecine générale (DRMG);

- deux planificateurs représentants la Régie régionale (RRSSS-03);

- trois administrateurs et représentants des instances locales;

- deux représentants de la Direction de santé publique de Québec (Équipe organisation des services et évaluation de la DSPQ);

+ un professionnel des services infirmiers;

- une personne externe impliquée dans le soutien à l'organisation des services sur le territoire et ayant siégé à la table.

Les informations colligées proviennent des verbatims des 12 entrevues et des PV des rencontres de la TTO et les rapports d'activités soumis par certains groupes d'acteurs. Une triangulation a été réalisée avec l'information fournie dans la documentation produite au sein du territoire, de la Direction régionale de la santé publique $\left(\mathrm{DSPQ}^{2}\right)$ et de la Régie régionale des Services de santé et services sociaux de la région 03 (région de Québec) (RRSSS-03) et du rapport final des travaux de la TTO déposé auprès de la RRSSS-03 par la TTO. L'analyse de l'information extraite a été réalisée conformément aux dimensions conceptuelles précisées dans ce qui précède.

\section{Résultats}

Les résultats sont présentés selon une logique chronologique et respectent les dimensions relatives aux cadres conceptuels retenus aux fins de cette étude. Le temps 0 , ou archétype 1, représente la période avant l'implantation de la TTO; la période intermédiaire couvre la période d'implantation et de fonctionnement de la TTO; le temps 1 , ou archétype 2, est celui du dépôt du rapport final des travaux de la table et finalement une présentation des conséquences.

Temps 0 : Avant l'implantation de la TTO, l'architecture organisationnelle en vigueur sur le territoire offrait un cadre de réalisation formel pour les activités relevant du mandat de chacun des groupes d'acteurs sans égard pour la formalisation des liens entre les deux secteurs. En effet, peu dans ce que décrivent les plans d'organisation des services de la RRSSS-03 était explicite quant aux activités et aux conditions reliées à la réalisation de l'interface SP-SCPL. Seuls les CLSC, dans le cadre de leur mandat de SP, se devaient de développer des activités de collaboration avec la SP. Néanmoins, ces collaborations ne représentaient qu'une faible proportion des activités de première ligne réalisées dans la région.

2 Dans le texte l'acronyme SP réfère à la DSPQ. 
Développement d'interfaces entre les services de santé publique et les services cliniques de première ligne à Québec

Au sein de cette configuration organisationnelle, presque tous les représentants des groupes d'acteurs interviewés sont satisfaits de l'adéquation entre la SO dans laquelle ils évoluaient et la conception qu'ils avaient de leur rôle par rapport à la perspective populationnelle. Seuls ceux de la SP ressentaient une certaine incohérence entre la configuration organisationnelle et leurs SI. En effet, quoique satisfaits par rapport aux activités dites traditionnelles comme la vaccination, ils déplorent un manque d'engagement des cliniciens dans d'autres activités moins traditionnelles comme la réduction des iniquités. En effet, ils considèrent que la SO dans laquelle la SP sinscrit offre peu de cadres formels pour le développement de l'interface. Les représentants de la SP étaient en situation de non-convergence avec ceux des autres groupes d'acteurs. Du point de vue organisationnel, les représentants de la SP déplorent le manque de coordination et de structuration des activités visant de manière spécifique l'interface. Du point de vue des SI, ils soulèvent la question de l'absence de responsabilité claire pour la perspective populationnelle et la faible implication des autres acteurs dans l'offre des services dédiés à des sous-groupes populationnels marginaux, tels les itinérants. Ces éléments laissent entrevoir une instabilité organisationnelle par rapport à la question du développement de l'interface SP-SCPL.

Limplantation de la TTO représente dès lors un événement marquant dans l'histoire du territoire. En effet, la TTO est un espace privilégié de concertation et de confrontation car il réunit autour d'une même table des acteurs professionnels et institutionnels d'appartenance diverse. Ce premier regroupement forme une coalition fonctionnelle, vu l'assiduité de la présence des différents acteurs (vérification de la liste de présence sur les PV des rencontres de la TTO). Néanmoins, il faut noter que chacun des acteurs était là avant tout pour défendre ou faire prévaloir les intérêts du groupe qu'il représente et les intégrer au plan d'organisation final.

Les négociations autour de la TTO font appel à une série de transactions, où chacun engage des ressources tout en évaluant les bénéfices potentiels qu'il va en tirer. Les liens individuels et collectifs sont mis à contribution pour rallier les acteurs et aboutir à des compromis parfois avec l'aide des pouvoirs administratifs, hiérarchiques ou corporatifs lorsqu'un consensus ne peut être atteint. Cependant, c'est essentiellement autour de préoccupations se rapportant au partage des tâches concernant la prise en charge des usagers, et non pas de la population, que se situent les débats. C'est un mouvement parallèle qui à ses débuts est extrêmement marginal et discret. Ce mouvement est conduit par les deux représentants de la SP. Ils essaient dans cette mouvance de saisir l'occasion pour susciter un intérêt de la part de tous les membres de la TTO pour l'interface SPSCPL. Ces acteurs profitent de ce que les cliniciens remettent en question leurs responsabilités et manières de faire, pour les inciter à étendre leur champ de questionnement à des préoccupations d'ordre également populationnel. Par cette approche, la SP vise l'appropriation par les cliniciens de l'idée qu'il leur incombe désormais une responsabilité pour une population et non pas seulement pour une clientèle. Le but recherché par 
la SP est l'appropriation par les cliniciens de l'idée qu'une organisation des services cliniques doit tenir compte du besoin d'une interface fonctionnelle SP-SCPL. Naît alors une coalition, formée par la SP et le CLSC, qui stratégiquement sengagent à travailler non seulement à mettre en avant leurs préoccupations mais aussi à défendre celles des cliniciens, et ce afin de les rallier autour de leurs intérêts d'ordre populationnel. C'est ainsi que graduellement la coalition SP-CLSC acquiert une reconnaissance et une légitimité de la part des cliniciens. Dès lors les représentants de la SP et celui du CLSC prennent le leadership de la rédaction du rapport final de la TTO.

Temps 2 : représente le moment du dépôt du rapport final de la TTO. Ce rapport est le résultat d'un consensus sur le réaménagement structurel à mettre en place, et dans lequel les différents groupes d'acteurs représentés auprès de la TTO se partagent les activités cliniques minimales à offrir à la population. De façon remarquable, la proposition accorde explicitement une place à l'interface SP-SCPL. La proposition soutient le besoin d'associer la SP à l'ensemble des travaux qui se réaliseront à l'avenir et de lui accorder le leadership pour déterminer les populations vulnérables pour lesquelles une attention particulière doit être portée. Même si les cliniciens ne souhaitent pas sinvestir dans le développement de l'interface, ils sont devenus plus conscients de l'importance, autant pour leur clientèle que pour la population générale, d'avoir des liens formels et fonctionnels avec la SP. Un changement majeur des valeurs et croyances des cliniciens sest donc opéré quant à leur responsabilité face au développement de l'interface SP-SCPL. Les travaux de la TTO ont permis une conscientisation des acteurs par rapport à la responsabilité collective vis-à-vis de la population et la nécessité de développer l'interface pour pouvoir mieux y répondre.

Les conséquences substantives du processus entourant les travaux de la TTO sont exprimées à travers la proposition de l'introduction de deux objectifs sur dix du rapport final de la TTO, directement en lien avec la perspective populationnelle. Ces propositions concernent la mise en place d'un continuum de services entre SP, responsable de l'identification des populations vulnérables, et CLSC, responsable de la prise en charge de ces populations. Cependant, on remarque que les démarches entreprises au début du processus par la SP font appel à des principes de collaborations et d'ententes mutuelles entre tous les acteurs, que seul le CLSC intègre de manière explicite et opérationnelle. Les autres acteurs cliniques, quant à eux, ne partagent pas forcément les valeurs véhiculées par la SP et ne simpliquent pas vraiment dans la formalisation de l'interface SP-SCPL qu'ils jugent être du ressort du CLSC. Néanmoins, le gain majeur pour la SP réside dans le fait que tous les acteurs de la première ligne sefforcent de fonctionner en réseau et ne rejettent pas le principe d'une première ligne conçue selon une approche populationnelle, plutôt qu'axée sur les clientèles. Les conséquences symboliques sont relatives à l'image que veut véhiculer le territoire, à savoir celle d'un espace où la concertation intersectorielle a réussi. Cette image est d'ailleurs renforcée par le fait que l'ensemble des membres de la TTO décide de la maintenir 
même après la fin des travaux. Cette décision est stratégique pour la SP, car cette table offre une occasion pour maintenir l'appropriation de la perspective populationnelle par les cliniciens. Elle est donc un atout pour le développement de l'interface. Les conséquences politiques sont essentiellement la reconnaissance par les cliniciens de la légitimité de la SP pour śimpliquer dans le processus d'organisation des services cliniques de première ligne.

\section{Discussion et conclusion}

Le jeu des acteurs s'interprète principalement au moyen des stratégies qu'ils développent pour faire prévaloir leurs attentes, protéger leurs acquis et maximiser leurs bénéfices. La dynamique qui en résulte est étroitement dépendante de l'atteinte d'un équilibre entre les stratégies pour et celles contre le changement. Sur le territoire à létude, la préoccupation pour le développement de l'interface SP-SCPL a été principalement le fait des représentants de la SP. Cet intérêt est à la base des stratégies que développeront ces acteurs au sein de la TTO pour atteindre leur objectif : l'adhésion des acteurs autour de la TTO à la perspective populationnelle et le déclenchement d'initiatives de formalisation de l'interface.

Les représentants de la SP qui se sont tout d'abord assurés de l'adhésion de leur allié sur le terrain, le CLSC, ont développé des stratégies susceptibles d'influencer le cours des événements et la rédaction du rapport final. Dans le contexte étudié, le développement d'une interface SP-SCPL a nécessité la formation d'une coalition qui lui était dédiée spécifiquement. La création de cette coalition a nécessité un leadership organisationnel ayant un réel impact sur l'orientation du processus transformationnel des SCPL. Seule la SP a pu assumer ce leadership et mener cette coalition, car, plus que les autres, elle y voit un intérêt qui correspond à sa raison d'être.

Ces constats viennent confirmer que deux aspects majeurs, à savoir le leadership de la SP et la gestion du changement en première ligne, sont à la base même du développement de l'interface SP-SCPL, éléments rapportés dans la littérature (Lasker 1997; Weiss et al. 2002; Wilson 2002; Ferrari et Rideout 2005). L'ajout que représente notre contribution à l'avancement des connaissances est d'une part la mise à contribution du leadership de la SP dans le façonnement du cours de l'implantation d'un changement organisationnel, ce qui a peu été documenté auparavant. D'autre part, nos constats montrent de manière opérationnelle comment le jeu des acteurs est une composante déterminante de l'évolution d'un changement organisationnel. La particularité de notre étude se cantonne au choix de notre unité d'analyse : une TTO à Québec. Cette modalité de fonctionnement imposée par une initiative régionale a offert aux acteurs du terrain un espace de confrontation et de négociation ayant permis la prise de décisions collectives basées sur le consensus. Consensus ayant entraîné une contribution équitable et des gains équilibrés pour tous les acteurs en présence. 
Cette constatation nous permet de conclure que la négociation et la confrontation des acteurs sont un moyen efficace pour l'atteinte d'un consensus organisationnel pour le développement de l'interface mais que les moyens et les stratégies à engager restent encore à développer et que jusquà présent peu de conclusions permettent d'offrir des outils valides et efficaces pour atteindre ce consensus.

$\mathrm{Au}$ Québec, la réforme actuelle offre une occasion de taille pour le développement de l'interface SP-SCPL. En effet, l'implantation des réseaux locaux de santé (RLS) introduit des changements autant d'un point de vue paradigmatique que structurel et touche autant le système de santé en général que les différents niveaux d'offre de services et les pratiques professionnelles. Les fondements des RLS reposent sur la hiérarchisation des services et la responsabilité populationnelle. Ces deux fondements supposent un système de santé centré sur une première ligne soutenue par une deuxième et une troisième ligne, le tout configuré selon une approche globale de la santé. Ainsi, la première ligne se voit désormais attribuer non seulement des tâches dans la coordination de l'ensemble des services requis pour les clientèles, allant de la prévention à la réadaptation, mais aussi une responsabilité populationnelle. De plus, une situation relativement nouvelle pour tous les acteurs de terrain, celle de la mise en avant de l'imputabilité (sectorielle, organisationnelle et professionnelle) qui découle de la responsabilité populationnelle. Cette imputabilité représente pour la SP une occasion considérable pour formaliser des ententes avec les SCPL, notamment à travers les projets-cliniques. Alors, dans quelle mesure la SP sera-t-elle capable de jouer un rôle clé au sein de cette mouvance organisationnelle afin de développer des interfaces formelles avec les SCPL?

Veuillez adresser toute correspondance à : Jalila Jbilou, Université Laval, Département de médecine sociale et préventive, Pavillon de l'Est, 2180, Chemin Sainte-Foy, Québec, QC, Canada G1V 1S4, Téléphone : (418) 656-2131, poste 4261, Télécopieur : (418)656-7759, Courriel : fsajjb@hermes. ulaval.ca

Remerciements : les auteurs souhaitent remercier le Centre de recherche Saint-François-d’Assise de Québec pour le financement de la présente étude, dans le cadre d'une bourse de recherche à la maîtrise (2002-2003).

\section{BIBLIOGRAPHIE}

Adami, H.O., N.E. Day, D. Trichopoulos, et W. C. Willett. 2001. « Primary and secondary prevention in the reduction of cancer morbidity and mortality ». European journal of cancer 37 Suppl. 8 : S118-27.

Brandt, A.M., and M. Gardner. 2000. «Antagonism and accommodation: interpreting the relationship between public health and medicine in the United States during the 20th century ». American journal of public bealth 90(5) : 707-15.

Cameron, R., R. Walker, et M. Gough, P. McDonald. 2000. « Linking public health science and 
Développement d'interfaces entre les services de santé publique et les services cliniques de première ligne à Québec

practice: an example from the Canadian Heart Health Initiative ». Leadership in health services 13(4) : i-vii.

Commission sur l'exercice de la médecine des années 2000. 1998. Nouveaux défis professionnels pour le médecin des années 2000. Québec : Collège des Médecins du Québec: 300 pages.

Denis, J.-L., A. Langley, et L. Cazale. 1996. « Leadership and strategic change under ambiguity ». Organization studies 17(4):675-99.

Eastcott, H.H. 1982. «The total care of the arteriosclerotic patient ». Annals of the Royal College of Surgeons of England 64(1) : 13-19.

Elster, A.B., et C.M. Callan. 2002. «Physician roles in medicine-public health collaboration - future directions of the American Medical Association ». American journal of preventive medicine $22(3): 211-13$.

Ferrari, A., et B. Rideout. 2005. «The collaboration of public health nursing and primary care nursing in the development of a nurse managed health center ». Nursing clinics of North America 40(4) : 771-8.

Friedberg, F. 1993. Le pouvoir et la règle : dynamique de laction organisée. Paris : Seuil.

Frumkin, H. 2000. "Occupational and environmental medicine and primary care ». Primary care 27(4) : 813-30.

Gamson, W. 1961. «A theory of coalition formation. » American sociological review $26: 373-82$.

Glick, M. 2002. «Screening for traditional risk factors for cardiovascular disease: a review for oral health care providers ». Journal of the American dental association 133(3) : 291-300.

Greenwood, R., and C.R. Hinings. 1988. «Organizational design types, tracks and the dynamics of strategic change ». Organization studies $9(3): 293-316$.

Grunfeld, E., A. Gray, D. Mant, P. Yudkin, R. Adewuyi-Dalton, D. Coyle, D. Cole, J. Stewart, R. Fitzpatrick, et M. Vessey. 1999. «Follow-up of breast cancer in primary care vs specialist care : results of an economic evaluation ». British journal of cancer 79(7-8) : 1227-33.

Hawk, E.T., et S.M. Lippman. 2000. «Primary cancer prevention trials ». Hematology/oncology clinics of North America 14(4) : 809-30.

Hinings, C.R., et R. Greenwood. 1988. The dynamics of strategic change. Oxford : Basil Blackwell. Jbilou, J. 2004. Le défi de la transformation des services de 1ère ligne dans un territoire de Québec: une réorganisation initiée par les acteurs de terrain autour d'une vision introduite par les planificateurs. Département de médecine sociale et préventive. Québec: Université Laval: 106 pages.

Lasker, R. 1997. Medicine and public health : the power of collaboration. New York : New York Academy of Medicine.

Lasker, R.D., E.S. Weiss, et R. Miller. 2001. « Partnership synergy: a practical framework for studying and strengthening the collaborative advantage ». Milbank quarterly 79(2): 179-205.

Lévesque, J.-F., et P. Bergeron. 2003. « De l'individuel au collectif : une vision décloisonnée de la santé publique et des soins ». Ruptures 9(2):73-89.

Reinharz, D. 1999. Désinstitutionalisation. Département de médecine sociale et préventive. Montréal : Université de Montréal : 195 pages.

Reinharz, D., A.P. Contandriopoulos, et A.D. Lesage. 2000. «Organizational analysis of desinstitutionalization in a psychiatric hospital ». Canadian journal of psychiatry 45(6) : 539-43. 
Shi, L., B. Starfield, R. Politzer, et J. Regan. 2002. "Primary care, self-rated health, and reductions in social disparities in health $»$. Health services research 37(3):529-50.

Storm, H.H., et J. Olsen 2002. « Prevention of cancer : what do we do in Denmark and what can we achieve? ». Ugeskr Laeger 164(22) : 2876-81.

Visscher, T.L., et J.C. Seidell. 2001. "The public health impact of obesity ». Annual review of public health $22: 355-75$.

Weiss, E.S., R.M. Anderson, et R.D. Lasker. 2002. « Making the most of collaboration : exploring the relationship between partnership synergy and partnership functioning ». Health education and behavior 29(6) : 683-98.

Wilson, J.L. 2002. « Leadership development : working together to enhance collaboration ». Journal of public health management practise $8(1): 21-6$.

Wright, P.F. 1995. "Global immunization: a medical perspective ». Social science and medicine $41(5): 609-16$.

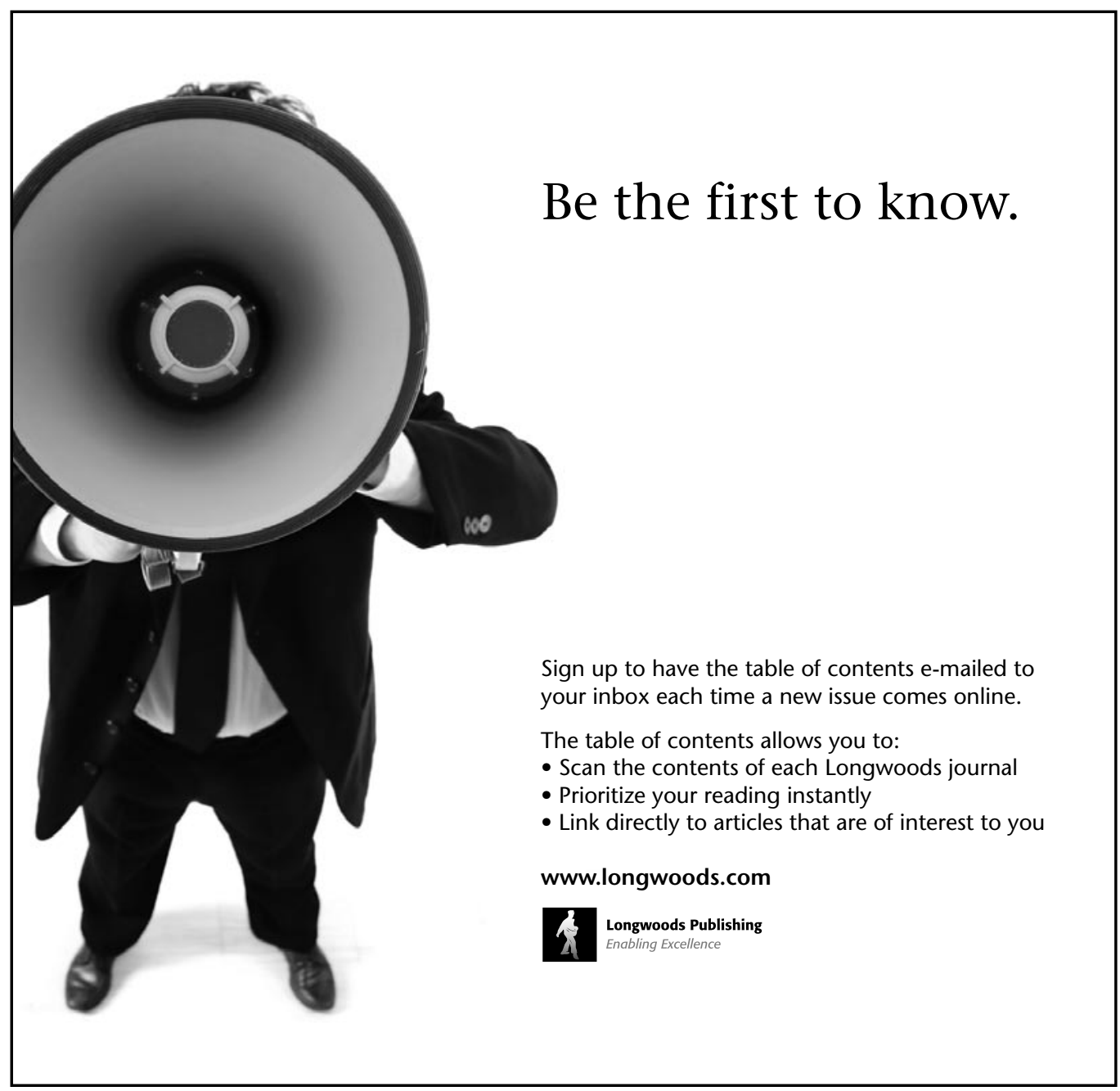

